\section{The Times They Are A'Changin': Changing with the Times}

\author{
"Nothing is permanent but change" (Heraclitus, 500 BC).
}

"The single greatest power in the world today is the power to change. . . . The most recklessly irresponsible thing we could do in the future would be to go on exactly as we have done in the past. . . ."(Karl W. Deutsch, 1977).

In keeping with a 65-year-old tradition, The Journal of Clinical Investigation (JCI) will change hands again in March 1992, moving its editorial offices to La Jolla, California. The selection of the new editor and editorial board followed a nationwide search, in which detailed applications were required of prospective candidates. A beneficial consequence of this process was that it stimulated forethought by the incoming editors about changes that might be made in the direction of the Journal.

Over the last five years, the current editors have made many useful and creative modifications in the running of the JCI, and in its overall format $(1,2)$. The challenge before the La Jolla group was to plan changes that might be made in the future, to enhance the functioning and image of the JCI, without risking or compromising its present successful status. In considering the possibilities, we have sought the advice of current and past editors, Society members, and a sampling of reviewers, authors, and readers. Remarkably, there was near consensus about the areas that need particular attention, and about those that do not need change.

The JCI publishes high quality work in all aspects of human and mammalian biology and pathobiology. As judged by citation rate and overall impact, the Journal remains in the top $1 \%$ of all scientific publications $(3,4)$. The $\mathrm{JCI}$ is well known for its thorough and critical reviews of submitted manuscripts. However, many authors regard the review process as too slow, and exciting findings that need rapid review are often submitted elsewhere. This image actually lags behind the reality. The Bay Area editors have significantly streamlined the review process over the past few years (2). However, the JCI has also become a victim of its own success, and a significant stumbling block to further improvement is the increasing volume of manuscripts being received (see Fig. 1). As a nonprofit publication with limited space, the Journal now has an acceptance rate of less than $30 \%$, with projected rates for the future being even lower. Thus, new approaches need to be developed to better serve submitting authors and readers.

Several changes are planned to improve the speed and efficiency of the review process, without compromising quality. To ensure broader expertise and more efficient service, the editorial board will have a larger number of associate editors. To avoid long drawn-out negotiations that exhaust authors, editors, and reviewers, evaluation of revised manuscripts will be limited to a single cycle. Other measures include a uniform submission form (see this issue), further computerization of manuscript tracking, liberal use of fax and overnight mailing services, and exploration of electronic publishing. However,

J. Clin. Invest.

(c) The American Society for Clinical Investigation, Inc.

$0021-9738 / 92 / 03 / 0721 / 02 \$ 2.00$

Volume 89, March 1992, 721-722 even with all of these measures, the timely review of such a large number of manuscripts remains a formidable challenge. Current and past editors indicate that a substantial fraction of submissions are recognizable at the outset as having no hope of achieving a publishable priority. Ultimately, it would be best for all concerned (authors, reviewers, and editors) if such manuscripts could be rapidly and fairly identified and returned without a prolonged and detailed review. Such "prescreening" is currently practiced by some other prestigious journals, with varying degrees of success and fairness (depending upon one's point of view as an editor, successful author, or rejected author). Our challenge was to design a prescreening mechanism for the JCI that would be efficient and rapid, without compromising the fairness and objectivity that must be part of the review process (5). Based upon much thought and consultation, the approach described below will be tried out as soon as it is feasible, sometime during 1992.

A small minority of incoming manuscripts will be screened out by the editors and returned unreviewed because of fatal flaws, e.g., inappropriate content, ethical issues, etc. All others will be assigned to an associate editor, who will send each out immediately to two expert reviewers. They will be asked to screen the manuscript immediately and assign a priority based on content, originality, quality, relevance, and interest. If the manuscript is considered to be in the lower half overall, a form will be promptly faxed back by the reviewer, with brief written comments. Meanwhile, the associate editor will have formed an independent opinion. If at least two of the three individuals independently feel that the manuscript is unlikely to achieve a publishable priority, it will be returned to the author with some reasons for the negative decision. However, if at least two of the three feel that the manuscript merits further consideration, the review will proceed without prejudice regarding the initial screening decision. Thus, manuscripts that pass this initial screening will already be in the hands of expert reviewers, and should have a reasonable likelihood (about one in two) of eventual acceptance. While no screening system can be perfect, this approach has several advantages. First, each manuscript will have been screened independently by three individuals: two expert reviewers and an associate editor. Second, a single negative opinion at screening will not prevent the manuscript from being finally accepted. Third, the overall work load of the reviewers and editors will be reduced, allowing them to deal more efficiently with manuscripts that do merit complete review. Fourth, the initial screening process will not delay the review of the eventually successful manuscript; rather, it may even speed it up, because the immediate prescreening ensures that the reviewer takes a first look at the manuscript soon after receipt. Finally, expert reviewers tend to be advocates of their own fields. Thus, a manuscript of high quality is less likely to be turned away simply because someone feels that it is not "sexy" or "popular" enough in content. Overall, we hope that this process will deal with many of the identified problems, while still preserving the best qualities of the original peer review system (5).

The traditional role of the $\mathrm{JCI}$ has been to publish the best research in human biology and physiology, and investigation relevant to human disease. In recent years, the inclusion of related work in mammalian biology has appropriately expanded the scope of the Journal. We are now in the midst of an unprecedented explosion of knowledge in human and mammalian biology, that has direct relevance and applicability to 


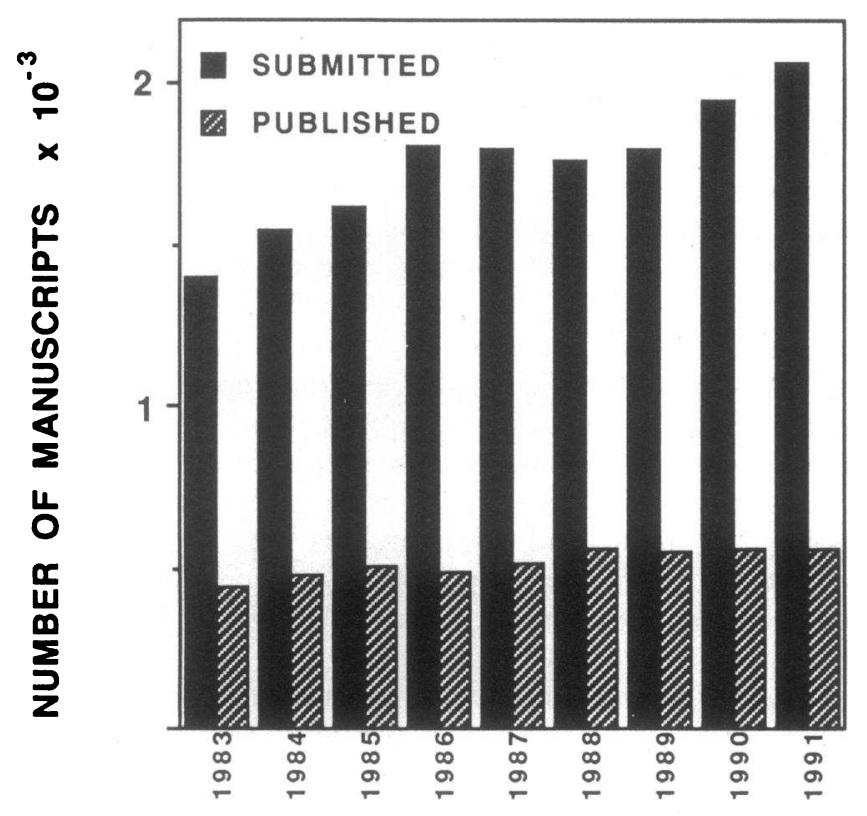

Figure 1

"clinical investigation." In many cases, human biology has become the paradigm for understanding the biology of lower organisms, rather than vice versa. Thus, the boundaries between basic and clinical investigation are becoming increasing blurred. Some would argue that despite the efforts of current and past editors, the JCI is facing an increasing challenge in maintaining its competitive edge in this exciting new frontier. Why should this be? In times past, this type of research was usually performed by "clinical investigators" who were medical scientists based in medical schools, often in clinical departments. However, in recent years, there has been a substantial increase in the number of "basic" researchers taking a leading role in studying human biology and pathobiology. By prior tradition, the cutting-edge work of such scientists is frequently published in other general or specialty journals, rather than in the JCI. Our goal in the next five years is to attract submissions of the very best work from investigators of all backgrounds who are interested in human biomedical research. We hope to do this by improving the handling of manuscripts as described above, by involving basic researchers in the editorial board, and by making the JCI an attractive place to publish novel and exciting new advances.

The JCI has previously been well-served by a national editorial committee drawn from the ranks of the Society. This tradition will continue. However, to ensure even broader coverage and expertise in all areas, an expanded board of consulting editors is planned (a full listing is presented in the masthead of this issue). These individuals will serve many functions, including the provision of advice to the editors, as well as prescreen- ing and reviewing of some manuscripts. In constituting this board, we have drawn somewhat heavily upon expertise from the many research institutions of Southern California. The reasons for this are purely logistical, and have to do with maximizing the efficiency of the review process. A unique feature of the $\mathrm{JCI}$ is that the editor and associate editors meet on a weekly basis to discuss all reviewed manuscripts, and to render group decisions on them. To make this possible, all these individuals must also be geographically clustered in one area. This, along with the number of consulting editors from the UCSD School of Medicine, could raise concerns of provincialism or bias. To avoid the possibility of favoritism, all manuscripts submitted by UCSD authors will be handled by guest editors from outside the institution, with appropriate steps to assure uniformity in the speed and quality of the review process.

The deliberate broadening of the content of the JCI over the last decade has resulted in decreased representation for some of its more traditional readers, particularly those studying whole organ and whole animal physiology and metabolism. This is an inevitable consequence of the ongoing revolution in cellular and molecular medicine. However, it will not be very long before human biology at the cellular and molecular level will meld directly with the study of specific organs and the whole organism. Thus, the JCI must preserve a balanced representation of all facets of research relevant to "human biology", from physiology to molecular biology. This can be achieved if excellence and originality are the major criteria for acceptance rather than trends and fashions.

Change of any sort is unsettling. Some of the proposed changes will probably raise concerns among some authors and readers. The editors plan to monitor and assess their impact carefully, and to make adjustments as necessary. Overall, we believe that the changes we will make in the running of the JCI will enhance the quality and appeal of the Journal. Meanwhile, we welcome any suggestions or comments that our readers and contributors may have.

\section{Ajit Varki UCSD School of Medicine for The Editorial Board}

\section{References}

1. Scharschmidt, B. F. 1990. Something old, something new, something blue. J. Clin. Invest. 85:1.

2. Scharschmidt, B. F. 1992. Change in editorial command. J. Clin. Invest. 89:1-2.

3. Science Citation Index. Journal Citation Reports. 1990. Institute for Scientific Information, Philadelphia.

4. Garfield, E. 1987. Fifty classics from the Journal of Clinical Investigation over 60 years of Nobel-class research. Curr. Contents. 8:3-8.

5. Wilson, J. D. 1978. Peer review and publication. J. Clin. Invest. 61:16971701. 